\title{
Authors' Reply to Katsuhiro Toda's Comment on “Proton Pump Inhibitor Use and Risk of Developing Alzheimer's Disease or Vascular Dementia: A Case-Control Analysis"
}

\author{
Patrick Imfeld ${ }^{1,2} \cdot$ Michael Bodmer $^{3} \cdot$ Susan S. Jick ${ }^{4,5} \cdot$ Christoph R. Meier $^{1,2,4}$
}

Published online: 5 October 2018

๑) Springer Nature Switzerland AG 2018

\section{Dear Sir,}

From a purely statistical point of view we agree with Katsuhiro Toda [1] that an adjusted odds ratio (OR) (95\% confidence interval [CI]) of 1.18 (1.04-1.33) for long-term users of $\geq 100$ prescriptions for proton pump inhibitors (PPI) may reflect a 'statistically significantly' increased risk of developing vascular dementia (VaD). There are, however, some aspects that we would like to point out regarding the interpretation of this OR. First, in observational drug safety research, an OR of 1.18 in a large observational study is a somewhat crude estimate of the relative risk, as the recorded clinical data are never $100 \%$ correct and comprehensively recorded, not even in a high quality database such as the Clinical Practice Research Datalink (CPRD), encompassing over 10 million patient records [2]. Second, despite all efforts to eliminate confounding, there will always be some degree of residual confounding in any such analysis. In this case, the adjusted OR of 1.18 is substantially lower than the corresponding unadjusted OR of 1.74, which indicates that the unadjusted OR is confounded. Full adjustment for

This reply refers to the article available at https://doi.org/10.1007/ s40264-018-0740-5.

Christoph R. Meier

christoph.meier@usb.ch

1 Basel Pharmacoepidemiology Unit, Division of Clinical Pharmacy and Epidemiology, Department of Pharmaceutical Sciences, University of Basel, Basel, Switzerland

2 Hospital Pharmacy, University Hospital Basel, Spitalstrasse 26, 4031 Basel, Switzerland

3 Medical Clinic, Zuger Kantonsspital, Baar, Switzerland

4 Boston Collaborative Drug Surveillance Program, Lexington, MA, USA

5 Department of Epidemiology, Boston University School of Public Health, Boston, MA, USA all known and unknown parameters in our analysis, which is of course not possible, would have driven the adjusted OR further towards unity. Third, and this is maybe the most relevant point, we must interpret the adjusted OR of 1.18 in the context of the whole analysis. There was clearly no duration-response relationship in the analysis, as none of the adjusted ORs for all other user categories $(1-4,5-19,20-49$, and 50-99 prescriptions) was increased, and as there was no trend towards higher OR with increasing drug use. Thus, we keep interpreting our analysis as a clear null result, and we consider it highly unlikely that this one isolated increased adjusted OR indicates an increased risk of VaD in association with PPI use.

\section{Compliance with Ethical Standards}

Funding No sources of funding were used to prepare this letter.

Conflict of interest Patrick Imfeld, Michael Bodmer, Susan S. Jick and Christoph R. Meier have no conflicts of interest relevant to the content of this letter.

\section{References}

1. Toda K. Comment on "Proton pump inhibitor use and risk of developing Alzheimer's disease or vascular dementia: a casecontrol analysis". Drug Saf. 2018. https://doi.org/10.1007/s4026 4-018-0740-5.

2. Herrett E, Gallagher AM, Bhaskaran K, Forbes H, Mathur R, van Staa T, et al. Data resource profile: clinical practice research datalink (CPRD). Int J Epidemiol. 2015;44:827-36. 